\title{
Laser cleaning of polymer surfaces
}

\author{
T. Fourrier ${ }^{1}$, G. Schrems ${ }^{1}$, T. Mühlberger ${ }^{1}$, J. Heitz ${ }^{1}$, N. Arnold ${ }^{1}$, D. Bäuerle ${ }^{1, *}$, M. Mosbacher ${ }^{2}$, J. Boneberg ${ }^{2}$, P. Leiderer ${ }^{2}$ \\ ${ }^{1}$ Angewandte Physik, Johannes-Kepler-Universität, Linz, 4040 Linz, Austria \\ ${ }^{2}$ Universität Konstanz, Department of Physics, 78457 Konstanz, Germany
}

Received: 13 July 2000/Accepted: 14 July 2000/Published online: 9 November 2000 - @ Springer-Verlag 2000

\begin{abstract}
We have investigated the removal of small spherical particles from polymer surfaces by means of 193-nm $\mathrm{ArF}$ and 248-nm KrF laser light. Polystyrene (PS) particles with diameters in the range of $110 \mathrm{~nm}$ to $1700 \mathrm{~nm}$ and silica particles $\left(\mathrm{SiO}_{2}\right)$ with sizes of $400 \mathrm{~nm}$ and $800 \mathrm{~nm}$ are successfully removed from two different substrates, polyimide (PI) and polymethylmethacrylate (PMMA). Experiments were performed in air $\left(23{ }^{\circ} \mathrm{C}\right.$, relative humidity $\left.24 \%-28 \%\right)$ and in an environment with a relative humidity (RH) of about $90 \%$.
\end{abstract}

\section{PACS: $42.62 . \mathrm{Cf}$}

Laser cleaning of surfaces from particles with diameters in the range from $100 \mathrm{~nm}$ to several microns has become increasingly important in lithography [1], semiconductor device fabrication [2-6], micromechanics, optics, telecommunications, etc.

Conventional techniques such as ultrasonic and megasonic cleaning, wiping and scrubbing, high-pressure jet spraying, etching, plasma cleaning, etc., are often inadequate for the removal of particulate contaminants of micron and submicron size. The problems with these techniques include ineffectiveness, addition of contaminants, damage of prefabricated parts, etc. It has been demonstrated that laser cleaning can be used to efficiently remove micron and submicron particles from solid surfaces. Such particulates adhere with relatively strong forces [7] that are difficult to overcome with the traditional cleaning techniques. Laser cleaning based on the absorption of pulsed-laser radiation by the substrate and/or the particulates is denoted as dry laser cleaning (DLC) $[6,8]$. If the removal of particulates is related to the superheating and explosive evaporation of an auxiliary thin liquid film, the technique is denoted as steam laser cleaning (SLC) $[4,5]$.

Since 1991 researchers have studied the removal of particles mainly from silicon wafers. This has provided theoretical modeling for what are considered the main forces involved in cleaning [1] as well as describing the detachment

*Corresponding author.

(Fax: +43-732/2468-9242, E-mail: dieter.baeuerle@jk.uni-linz.ac.at) of the particles from the surface and their spatial distribution after leaving the substrate in relation to the surface roughness [9]. In recent publications there has been a significant increase in the use of polymer science in the fields of microelectronics and sensor technology [10]. From polymers used in packaging to polymers used as electrets, polymers will soon be facing the same cleaning difficulties as conventional semiconductor materials. Only a few existing papers [11,12] describe the removal of residues of polymers from polymers. Nonetheless, to the authors' knowledge, no papers have dealt with laser-induced removal of particles from polymer substrates.

In this paper we investigate laser cleaning of PI (polyimide) and PMMA (polymethylmethacrylate) from PS (polystyrol) and $\mathrm{SiO}_{2}$ (silica) microspheres of different sizes.

\section{Experimental procedure}

The experimental setup consists of a $\mathrm{KrF}$ excimer laser (Lambda Physik LPX 205), which provides at $\lambda=248 \mathrm{~nm}$ a maximum pulse energy of $E_{\ell} \approx 470 \mathrm{~mJ}$ (at a $v_{\mathrm{r}}=1 \mathrm{~Hz}$ repetition rate). Its pulse length is $\tau_{\ell}(\mathrm{FWHM}) \approx 31 \mathrm{~ns}$ and its repetition rate is between $1 \mathrm{~Hz}$ and $30 \mathrm{~Hz}$. The pulse energy is computer-controlled by an external adjustable tunable dielectric attenuator.

The output from a circular aperture was imaged using a lens onto the polymer target to a spot of about $0.8 \mathrm{~mm}^{2}$. Due to the quasi-top-hat beam profile, the energy density remained constant over the irradiated region. The target was perpendicular to the plane of the horizontal incoming beam (Fig. 1).

Both polymer substrates were $50-\mu \mathrm{m}$-thick foils provided by Goodfellow Ltd. The PS and the $\mathrm{SiO}_{2}$ particles used as pollutants were supplied by Bangs Laboratory Ltd. The size distribution of the particles was specified to be within 1 and $10 \%$ of the average size.

Pollution of the sample was achieved via a spin-coating method [13]. A droplet of dense particles was rarefied in IPA (isopropanol). This solvent offered high evaporation rates and low concentrations of colloids and al- 


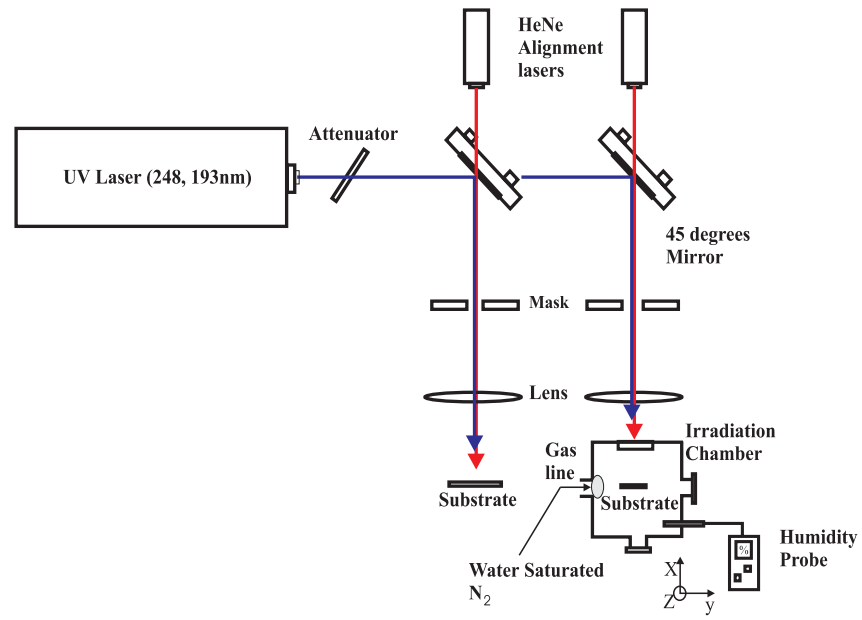

Fig. 1. Experimental setup

lowed a quasi-homogeneous distribution of particles on the surface. Each sample was visually checked by means of a microscope to verify the density of particles (average $N_{\mathrm{o}} \approx 2 \times 10^{5}$ particles $/ \mathrm{cm}^{2}$ ) and to limit the number of clusters present on each substrate. Care was taken to avoid agglomeration of particles, as it simplified the analysis to use a specific size of pollutant and allowed the detection of cleaning thresholds for various sizes. To limit the uncertainties in the measurements, two pictures taken exactly at the same location on the polymer surface prior to and after irradiation were collected by a video camera attached to a microscope and linked via a framegrabber to a computer. This resulted in two different sets of measurements: monitoring of the removal of the particles and monitoring of the eventual particle redeposition on the surface.

Finally, the technique relied on the accurate repositioning of the sample within the range of a few microns. This was found necessary to ascertain the irradiation of the preanalyzed areas. To do this all polymer substrates were fixed on a glass slide, held vertically in a moving plane perpendicular to the incident laser beam and allowed to slide in and out for further analysis. Two stepper motors controlled the movement of the sample holder. Particles were counted by using commercially available image analysis software.

Laser-pulse energies were varied from a few to tens of millijoules. In the higher-energy range $(>10 \mathrm{~mJ})$, fluences were measured with an accuracy of around $2 \%$. In the lowerenergy range, due to the pulse-to-pulse energy variation of the laser, the values were measured with an uncertainty close to $10 \%$. For these values, a systematic error was added to the energy measurements. This uncertainty was determined to be of the order of $3 \%$.

\section{Results and discussion}

Determination of the cleaning efficiencies as well as the cleaning threshold involves counting the number of particles per area prior to laser irradiation, $N_{\mathrm{o}}$, and after laser irradiation, $N$. For all our experiments, the cleaning efficiency is then defined as $\eta=\left(1-N / N_{\mathrm{o}}\right)$ and is later converted to a percentage. The number of pulses applied, if not specified, was set to $N_{\ell}=1$. The efficiency depends not only on the dif- ferent laser parameters such as the fluence, wavelength, and the number of shots, but also on the particles and substrate properties such as the absorption coefficients $\alpha$, thermal diffusivities, $D$, binding energies of the pollutants to the substrate, elastic constants, etc. In this respect, the results are specific to definite sets of experiments.

\subsection{Experiments at $248 \mathrm{~nm}$}

2.1.1 PI substrates. Three sizes of PS particles were used to characterize the removal of absorbing particles from an absorbing substrate (Table 1). It is important to accomplish cleaning without damage to the underlying material. For PI and $248 \mathrm{~nm}$, the fluence for surface modification was estimated as $\phi_{\text {sm }} \approx 20 \mathrm{~mJ} / \mathrm{cm}^{2}$. In our experiments no visible damage was observed in SEM pictures after 500 laser pulses with $17.5 \mathrm{~mJ} / \mathrm{cm}^{2}$, whereas such damage was observed with $\phi=22.5 \mathrm{~mJ} / \mathrm{cm}^{2}$. These data are in agreement with the single-pulse $(\mathrm{SP})$ ablation threshold $\phi_{\mathrm{th}} \approx 19 \mathrm{~mJ} / \mathrm{cm}^{2}$ reported in [14]. This threshold may actually correspond to the modification threshold $\phi_{\mathrm{sm}}$, as near-threshold mass loss is due to outdiffusion of volatile species $[15,16]$.

Particles with diameters of 800 and $320 \mathrm{~nm}$ were cleaned in SP experiments with efficiencies of $(69 \pm 5) \%$ and $(31 \pm 7) \%$, respectively, at a fluence slightly below $\phi_{\text {sm }}$, i.e. at $\phi \approx 17.5 \mathrm{~mJ} / \mathrm{cm}^{2}$. Figure 2 a shows that the thresholds for cleaning decrease with increasing particle size [1, 17]. For the case of 110-nm particles, the cleaning efficiency only reaches values below $(10 \pm 10) \%$ in the region below the modification threshold. As well as the removal, the characterization of small particles remains a problem. As the particle size gets smaller than a few hundred nanometres, optical microscopy becomes obsolete [18]. At this point, one requires the use of a scanning electron microscope, or alternative techniques such as light scattering from particles [19]. Thus, for very small particles, reliable data for cleaning become difficult to collect. This partially explains the very dispersed and scattered data points.

The removal of transparent $\mathrm{SiO}_{2}$ particles from PI (Table 1), as shown in Fig. 2b, is comparable to the cleaning of silicon wafers. In this case, SP particle removal is observed with an efficiency of around $(95 \pm 5) \%$ for both sizes of particles available. Although efficiencies are comparable, cleaning for the smaller particles starts later and requires about twice the fluence to reach comparable cleaning. This is expected from their different binding energies [7].

It is at this stage impossible to explain whether the reduced cleaning of $320-\mathrm{nm}$ PS particles relative to $400-\mathrm{nm}$ $\mathrm{SiO}_{2}$ particles from PI at fluences above $\phi_{\text {sm }}$ is due to changes in the binding energies, due to the higher elastic energy of

Table 1. Absorption coefficients for the different materials used in the experiments

\begin{tabular}{lcc}
\hline & $\alpha(248 \mathrm{~nm}) / \mathrm{cm}^{-1}$ & $\alpha(193 \mathrm{~nm}) / \mathrm{cm}^{-1}$ \\
\hline $\mathrm{PI}$ & $3.0 \times 10^{5}$ & $3.4 \times 10^{5}$ \\
$\mathrm{PMMA}$ & $130+70$ & $2 \times 10^{3}$ \\
$\mathrm{PS}$ & $6.5 \times 10^{3}$ & $8 \times 10^{5}$ \\
$\mathrm{SiO}_{2}$ & $<1$ & $<1$ \\
\hline
\end{tabular}



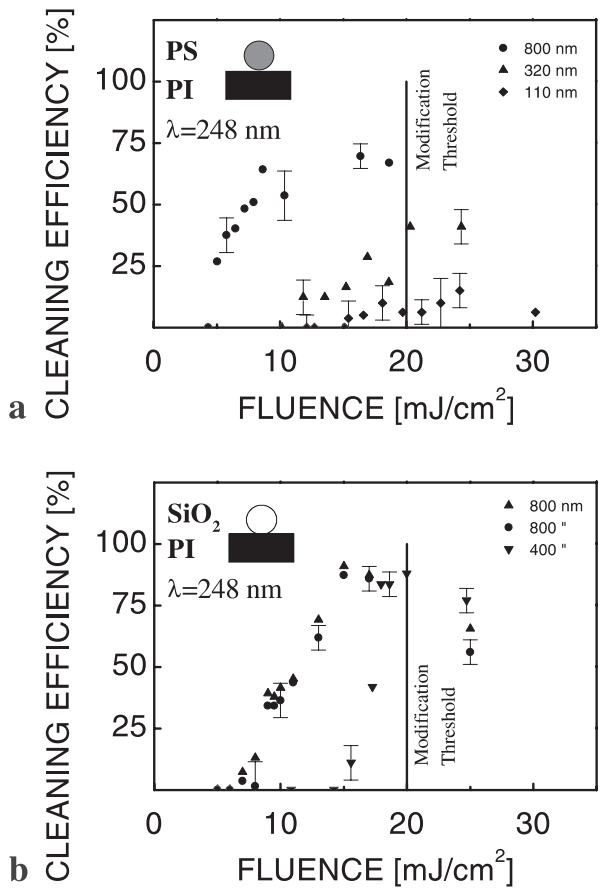

Fig. 2a,b. Single-pulse cleaning efficiency vs. laser fluence at $\lambda=248 \mathrm{~nm}$ for different particle materials and sizes. Substrate PI, RH 27\%: a PS particles; $\mathbf{b} \mathrm{SiO}_{2}$ particles

$\mathrm{SiO}_{2}$ with respect to PS, or due to other factors such as absorption and diffraction of light by the particles which can lead to focusing of light under the particles [20], etc.

2.1.2 PMMA substrates. PMMA is a poor light absorber at $248 \mathrm{~nm}$ (Table 1). The SP modification threshold is difficult to define due to incubation effects. We observed no visible morphology changes in SEM pictures after several laser pulses at fluences below $\phi_{\mathrm{th}} \approx 200 \mathrm{~mJ} / \mathrm{cm}^{2}$. This agrees with reported ablation thresholds which are between $\phi_{\text {th }} \approx 200 \mathrm{~mJ} / \mathrm{cm}^{2}$ [21] and $300 \mathrm{~mJ} / \mathrm{cm}^{2}$ [22]. Color changes (incubation) were observed with several pulses starting from $\phi_{\mathrm{sm}} \approx 140 \mathrm{~mJ} / \mathrm{cm}^{2}$. A cleaning efficiency greater than $80 \%$ was achieved for PS particles with a single pulse using fluences in the range $160 \mathrm{~mJ} \leq \phi \leq \phi_{\text {th }}$ (Fig. 3). Thus, in contrast to the case of polluted silicon substrates, where it was demonstrated that the acceleration/deceleration caused by the thermal expansion of the substrate is responsible for cleaning [23], in the present case the expansion of particles seems to be the dominant factor. This is supported by the fact that with a fluence of $160 \mathrm{~mJ} / \mathrm{cm}^{2}$, which was big enough to result in incubation in our experiments, no removal of transparent $\mathrm{SiO}_{2}$ particles from the substrate was observed. Removal of $\mathrm{Al}_{2} \mathrm{O}_{3}$ from $\mathrm{SiO}_{2}$ was reported in [24].

\subsection{Experiments at $193 \mathrm{~nm}$}

When the wavelength is decreased from $248 \mathrm{~nm}$ to $193 \mathrm{~nm}$, the optical penetration depth decreases by about a factor of 10 for PMMA, and stays almost constant for PI (Table 1).

2.2.1 PI substrate. Single-pulse cleaning of PI from 400-nm $\mathrm{SiO}_{2}$ particles was possible from $10 \mathrm{~mJ} / \mathrm{cm}^{2}$ onwards with efficiencies from less than $(10 \pm 10) \%$ at $10 \mathrm{~mJ} / \mathrm{cm}^{2}$ up to $(92 \pm 5) \%$ at $17.5 \mathrm{~mJ} / \mathrm{cm}^{2}$. This value was just below the threshold for surface modification in our experiments (no observable damage of the PI surface after 500 pulses). The discrepancy from the reported value $\phi_{\mathrm{th}} \approx 14 \mathrm{~mJ} / \mathrm{cm}^{2}$ [14] is probably due to the longer pulse duration of our ArF laser. Neither the particles nor the substrate showed major changes in absorptivity and absorption coefficients as compared to the 248-nm case. The cleaning threshold $\phi_{\mathrm{cl}}$ decreases somewhat more than the modification and ablation thresholds. For $400-\mathrm{nm} \mathrm{SiO} 2$ particles $\phi_{\mathrm{cl}}(\mathrm{PI}, 193 \mathrm{~nm}) \approx 11 \mathrm{~mJ} / \mathrm{cm}^{2}$, while $\phi_{\mathrm{cl}}(\mathrm{PI}, 248 \mathrm{~nm}) \approx 14 \mathrm{~mJ} / \mathrm{cm}^{2}$ (Fig. 2b). Naturally, efficiencies at a given fluence $\phi>\phi_{\mathrm{cl}}$ are improved as well, i.e. with $N_{\ell}=1$ at $15 \mathrm{~mJ} / \mathrm{cm}^{2}$ we find $\eta(193 \mathrm{~nm}) \approx 75 \%$ whereas $\eta(248 \mathrm{~nm}) \approx 10 \%$.

By increasing the number of pulses, higher efficiencies can be reached at lower fluences. For instance, with $N_{\ell}=5$ and $15 \mathrm{~mJ} / \mathrm{cm}^{2}$ cleaning close to $(92 \pm 5) \%$ was achieved. This is comparable to the efficiency obtained with $N_{\ell}=1$ at $17.5 \mathrm{~mJ} / \mathrm{cm}^{2}$. Cleaning efficiencies approaching $(80 \pm 5) \%$ were obtained with $N_{\ell}=15$ at $12.5 \mathrm{~mJ} / \mathrm{cm}^{2}$. This offers a margin between $\phi_{\mathrm{cl}}$ and $\phi_{\mathrm{sm}}$ large enough to almost avoid surface damage.

After a sharp cleaning increase with $N_{\ell}$, all curves tail off to approach a maximum efficiency, which is rather small for fluences near $\phi_{\mathrm{cl}}$. The maximum efficiencies reached with MP irradiation are $(40 \pm 7),(80 \pm 5)$ and $(95 \pm 5) \%$ at 10 , 12.5 and $17.5 \mathrm{~mJ} / \mathrm{cm}^{2}$, respectively (Fig. 4; note that the data in Figs. 4 and 6 are obtained with counting software only).

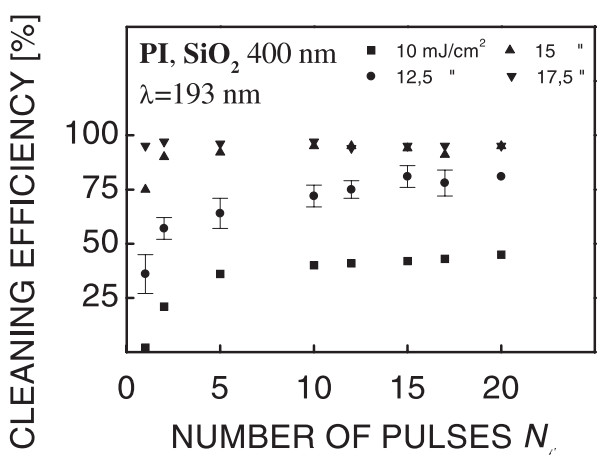

Fig. 4. Multiple-pulse cleaning efficiency for $\mathrm{SiO}_{2}(400 \mathrm{~nm})$ particles from PI at $\lambda=193 \mathrm{~nm}, \mathrm{RH} \sim 27 \%$
Fig. 3. Single-pulse cleaning efficiency of PS particles from PMMA. $\lambda=248 \mathrm{~nm}, \tau_{\ell}=29 \mathrm{~ns}, \mathrm{RH} \sim 28.4 \%$

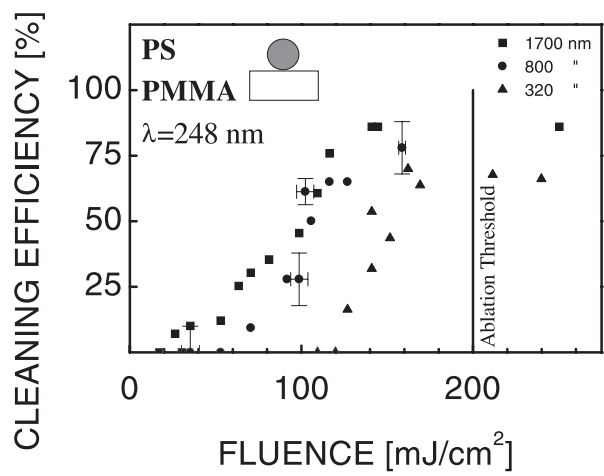


The results exhibit the main problem we have in dealing with polymers: the effective cleaning fluence and the modification fluence for the polymers are often close to each other.

2.2.2 PMMA substrates. The removal of $400-\mathrm{nm} \mathrm{SiO}_{2}$ particles from PMMA is less effective than from PI. The absorption coefficients of PMMA and PI differ by about a factor of 170 (Table 1). The modification/ablation thresholds are difficult to define due to incubation effects. In our experiments no morphological changes were observed in SEM pictures after a single pulse for $\phi<\phi_{\text {th }}$ (PMMA, $\left.193 \mathrm{~nm}\right) \approx 40 \mathrm{~mJ} / \mathrm{cm}^{2}$, which agrees with the reported MP value [25]. The cleaning threshold for these particles is $\phi_{\mathrm{cl}} \approx 18.5 \mathrm{~mJ} / \mathrm{cm}^{2}$ (Fig. 6). Single-pulse cleaning thresholds for different particles, substrates and wavelengths are summarized in Fig. 5.

Cleaning always improves with rising fluence as long as $\phi<\phi_{\mathrm{sm}}$. This is explained by the greater velocity acquired by the particles due to the stronger thermal expansion of the substrate with increased laser fluences. On each of the cleaning efficiency vs. fluence curves in the case of a single shot, one can observe three regimes. The first regime corresponds to $\phi<\phi_{\mathrm{cl}}$. In the second regime, cleaning increases steadily with increasing fluence. Subsequently, at higher fluences, cleaning slows down and finally may even decrease. The latter regime often occurs near $\phi_{\mathrm{sm}}$ where absorption and other physical and chemical characteristics of the polymer change.

As in the 248-nm case, at $193 \mathrm{~nm}$ saturation in the cleaning efficiency with respect to the number of shots is observed. An efficiency close to the maximum is reached for $N_{\ell}<10$ to 20 . This prevents an overload of pulses that may weaken in

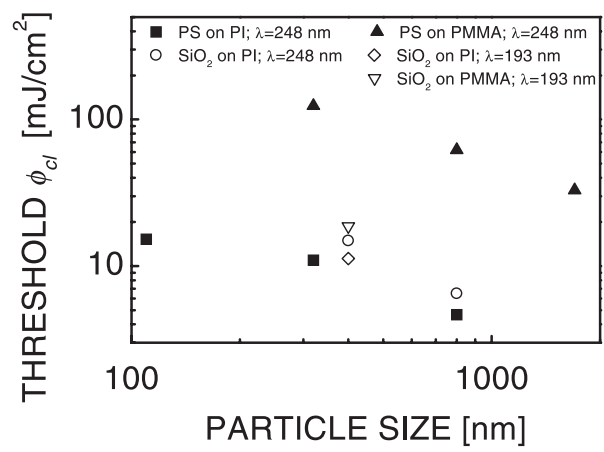

Fig. 5. Dependence of single-pulse cleaning threshold on particle size for different substrate and particle materials and irradiation wavelengths

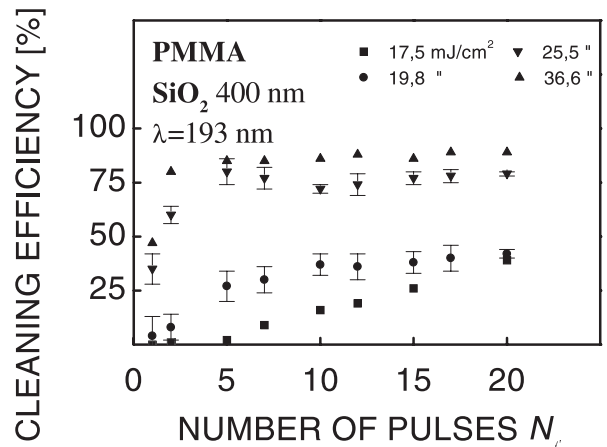

Fig. 6. Multiple-pulse cleaning efficiency for $\mathrm{SiO}_{2}(400 \mathrm{~nm})$ particles from PMMA at $\lambda=193 \mathrm{~nm}, \mathrm{RH} \sim 28.4 \%$ the long run the surface of the substrate. At $\phi \approx 25 \mathrm{~mJ} / \mathrm{cm}^{2}$, we obtained pollutant removal efficiencies of the order of $80 \%$ after five shots (Fig. 6). This fluence is smaller than the reported single-pulse threshold value $\phi_{\text {sm }}$ (PMMA, $193 \mathrm{~nm}$ ) $\approx 27 \mathrm{~mJ} / \mathrm{cm}^{2}$ [21] measured with a quartz crystal microbalance (QCM), which can be considered as the modification threshold.

As in the case of silicon, the repetition rate did not show any significant effect [17]. This should be expected as maximum thermal expansion is reached at the end of the nanosecond-laser pulse. The relaxation of this expansion is related to heat dissipation as a result of the volume and energy exchange with the ambient. This takes place within a few microseconds. Thus, to see the effect of the repetition rate, one would have to work at frequencies of the order of a few $\mathrm{kHz}$.

If we assume that the cleaning efficiency for a single pulse is given by $\eta \equiv \eta\left(N_{\ell}=1\right)$, in the ideal case of identical isolated particles the cleaning efficiency should increase with the number of shots according to

$\eta\left(N_{\ell}\right) \approx 1-(1-\eta)^{N_{\ell}}$.

Deviations from this simple law, which can be seen in Figs. 4 and 6, may be due to the different cleaning threshold fluences related to the size distribution of particles, the surface roughness of the substrate, etc.

For both wavelengths considered, experiments demonstrate, as commonly found in the literature on silicon in the case of DLC, an increase in the cleaning threshold $\phi_{\mathrm{cl}}$ with decreasing particle size (Fig. 5). This can be understood along the following lines. Upon expansion of the substrate, particles gain kinetic energies [1]:

$E_{\mathrm{kin}} \sim m v^{2} \sim \varrho_{\mathrm{p}} r^{3}\left(\frac{\beta_{\mathrm{T}} \phi_{\mathrm{a}}}{\varrho c_{\mathrm{p}} \tau_{\ell}}\right)^{2}$.

Here $m$ is the mass of the particle, $\varrho_{\mathrm{p}}$ is its density, and $r$ is its radius. $v$ is the expansion velocity, $\beta_{\mathrm{T}}$ is the (linear) coefficient of thermal expansion, $\varrho$ is the density and $c_{\mathrm{p}}$ is the specific heat of the substrate. $\phi_{\mathrm{a}}$ is the absorbed fluence and $\tau_{\ell}$ is the laser pulse duration. Cleaning will take place if this energy exceeds the adhesion energy. For the example of capillary forces, the adhesion energy is $E_{\text {ad }} \sim \sigma r^{2}$ where $\sigma$ is the surface tension coefficient. The situation may vary with other adhesion mechanisms [1]. In order to keep generality we write

$E_{\mathrm{ad}} \approx \delta_{n} r^{n}$,

where $1 \leq n \leq 2$. Here, $\delta_{n}$ is some proportionality coefficient. For example, $n=1$ corresponds to electrostatic double-layer adhesion and/or to Van der Waals (VdW) adhesion for a point contact and/or a contact area with radius $r_{d} \propto r^{1 / 2}$. The latter refers to plastic deformation. With $\mathrm{VdW}$ and elastic deformation one would expect $r_{d} \propto r^{2 / 3}$ and $n=5 / 3$ [26]. Using (2) and (3) the condition $E_{\text {kin }}>E_{\text {ad }}$ results in

$\frac{\beta_{\mathrm{T}} \phi_{\mathrm{a}}}{\varrho c_{\mathrm{p}} \tau_{\ell}}\left(\frac{\varrho_{\mathrm{p}} r^{3-n}}{\delta_{n}}\right)^{1 / 2}>1$ or $\phi \geq \phi_{\mathrm{cl}} \approx \frac{\varrho c_{\mathrm{p}} \tau_{\ell}}{\beta_{\mathrm{T}} A}\left(\frac{\delta_{n}}{\varrho_{\mathrm{p}}}\right)^{1 / 2} \frac{1}{r^{k}}$,

where $1 / 2<k \equiv(3-n) / 2<1$. Here, $A$ is the absorptivity of the substrate. $k=1 / 2$ corresponds, for example, to capillary adhesion, whereas $k=1$ corresponds, for example, to 
VdW with plastic deformation. Relationship (4) with $1 / 2<$ $k<1$ can tentatively explain the experimental observations in Fig. 5.

Clearly, such a treatment is oversimplified. Depending on the parameters (e.g. with longer pulses), the force balance may be more relevant than the energetic consideration [27]. Expansion of the substrate may lead to the compression of the particle and an increase in its elastic energy [28]. This energy is transformed into kinetic energy after the pulse, which results in cleaning. Elastic energy may significantly exceed the $E_{\text {kin }}$ value given by (2). This effect is less pronounced with smaller (lighter) particles, as they would rather accelerate as a whole than compress.

Thermal expansion of absorbing particles may also lead to an increase in both the kinetic and the elastic energy, even with a transparent (non-expanding) substrate. Besides, absorbing particles may be simply evaporated, provided $\phi \sigma_{\mathrm{p}}>$ $\varrho_{\mathrm{p}} \Delta H_{\mathrm{v}}^{\mathrm{p}} r^{3}$. Here $\sigma_{\mathrm{p}} \sim r^{2}$ is the particle absorption crosssection for $r \gg \lambda$ and $\sigma_{\mathrm{p}} \sim r^{3} / \lambda$ for $r \leq \lambda . \Delta H_{\mathrm{v}}^{\mathrm{p}}[\mathrm{J} / \mathrm{g}]$ is the evaporation enthalpy of the particle. If heat conduction into the substrate can be neglected, such a mechanism is more feasible for smaller particles.

Besides, local field enhancement under the particles was reported recently even for the particles smaller than the laser wavelength [29]. This can lead to a local ablation of substrate material, which facilitates particle removal. At the same time, this may result in substrate damage at fluences significantly below the modification threshold for unpolluted substrates. Clarification of the aforementioned questions may be a basis for further work.

As all experiments were performed with a beam spot of approximately $1 \mathrm{~mm}^{2}$, redeposition may be ignored. With larger spot sizes, however, redeposition may significantly decrease the cleaning efficiencies. This is currently under investigation.

\subsection{Increased relative humidity in the chamber}

2.3.1 PI substrates. Experiments were performed with 400and $800-\mathrm{nm} \mathrm{SiO}_{2}$ particles at $248 \mathrm{~nm}$. In this case only the substrate absorbs. When irradiated in an environment saturated with water vapor $\phi_{\mathrm{cl}}$ is $30 \%$ lower than under normal conditions (Fig. 7). For clarity only the results for 400-nmsize particles are shown, but a similar effect is observed at $800 \mathrm{~nm}$. In the case of increased relative humidity $(\mathrm{RH})$, the maximum efficiency is reached earlier, leaving a safer margin before modification of the substrate.

2.3.2 PMMA substrates. PS (320-nm) and $\mathrm{SiO}_{2}(400-\mathrm{nm})$ particles on PMMA substrates were irradiated with fluences similar to those used for cleaning under normal atmospheric conditions. Unlike in the case of PI, cleaning of PMMA showed neither improved efficiency nor a decrease in $\phi_{\mathrm{cl}}$ (Fig. 8). In the case of $\mathrm{SiO}_{2}$ no cleaning was observed. Although the RH in the irradiation chamber was increased, particles remained on the surface. As mentioned, PMMA has a very big absorption length at $248 \mathrm{~nm}$. In addition, water shows a very low absorptivity at this wavelength $(\approx 0.1$ at $248 \mathrm{~nm}$ ). The absorption of the particles is probably not sufficient to induce vaporization and facilitate their ejection from the substrate [30]. Thus, cleaning under "wet conditions"

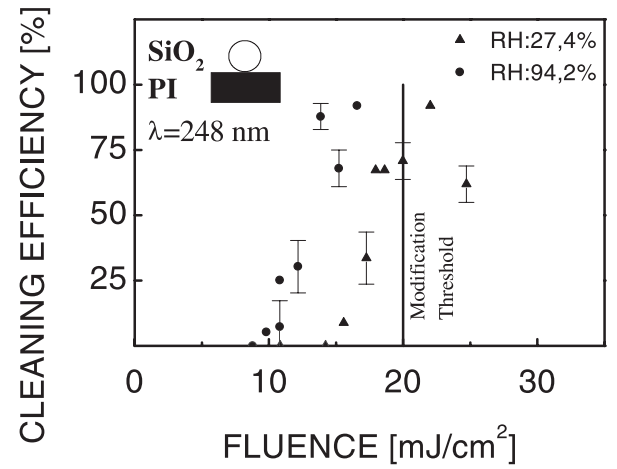

Fig. 7. Effect of relative humidity on removal of $\mathrm{SiO}_{2}(400 \mathrm{~nm})$ from PI $\left(N_{\ell}=1, \lambda=248 \mathrm{~nm}\right)$

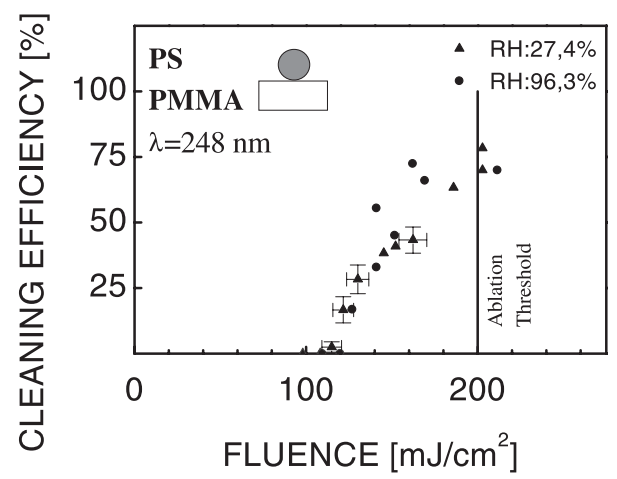

Fig. 8. Effect of relative humidity on removal of PS $(320 \mathrm{~nm})$ from PMMA $\left(N_{\ell}=1, \lambda=248 \mathrm{~nm}\right)$

seems to require strong substrate absorption to induce a noticeable change in the cleaning fluences.

Due to increased humidity, water condenses at the interstice between the particle and the substrate. Improved cleaning is most possibly initiated by the strong absorption of the substrate, which induces explosive evaporation and/or boiling of water, which takes the particles off the surface. Thus, cleaning with an increased RH becomes similar to the SLC method extensively studied $[5,6,13,19,31,32]$ for the case of silicon. The main advantage offered here is that this technique requires no direct liquid deposition on the surface, which may represent a technical difficulty or critical chemical invasion, especially in the case of polymers.

\section{Conclusions}

The present investigations demonstrate that it is possible to clean polymer surfaces with UV excimer laser light that is either absorbed by the substrate or the particulate. $\mathrm{SiO}_{2}$ particles of $400 \mathrm{~nm}$ and $800 \mathrm{~nm}$ on PI were removed with 193-nm and 248-nm radiation and single-pulse efficiencies of up to $(95 \pm 5) \%$.

Spherical PS particles with diameters down to $110 \mathrm{~nm}$ were cleaned off from both PI and PMMA substrates. The single-pulse efficiencies near the threshold of surface modification strongly decrease with particle size and they were only about $10 \%$ for the smallest particles. No cleaning was observed with (transparent) $\mathrm{SiO}_{2}$ particles deposited on (transparent) PMMA at $248 \mathrm{~nm}$. 
In contrast to silicon, the range of fluences between the cleaning threshold and surface modification of the polymer is rather narrow. Applying typically 10-20 pulses, one can improve efficiencies from $10 \%-50 \%$ up to $40 \%-90 \%$, depending on the experimental conditions. With a higher number of pulses, the cleaning efficiencies saturate.

Different cleaning mechanisms seem to be relevant:

- Rapid thermal expansion of the substrate. This favors removal of bigger particles on absorbing substrates.

- Particle expansion, which can remove strongly absorbing particles from transparent substrates.

- For very small particles with low vaporization enthalpy, evaporation of particles may become important.

Cleaning of a strongly absorbing substrate is usually more effective. However, depending on the parameters and the binding energies of the particle/substrate system, either of these other mechanisms may be responsible for cleaning.

Increased cleaning from absorbing substrates was shown for higher humidity. This suggests that such improved "dry" cleaning could be used for sensitive substrates as long as it reasonably absorbs the incoming light.

Acknowledgements. The authors acknowledge the financial support of the EU within the framework of the TMR project Laser Cleaning contract no. ERBFMRXCT98 0188.

\section{References}

1. D. Bäuerle: Laser Processing and Chemistry, 3rd edn. (Springer, Berlin, Heidelberg 2000)

2. J.D. Kelley, F.E. Hovis: Microelectron. Eng. 20, 159 (1993)

3. R. DeJule: Semicond. Int. 21, 64 (1998)

4. S.J. Lee, K. Imen, S.D. Allen: Appl. Phys. Lett. 58, 203 (1991)

5. W. Zapka, W. Ziemlich, A.C. Tam: Appl. Phys. Lett. 58, 2217 (1991)

6. A.C. Tam, W.P. Leung, W. Zapka, W. Ziemlich: J. Appl. Phys. 71, 3515 (1992)
7. R.A. Bowling: In Particles on Surfacesed, ed. by K.L. Mittal, Vol. 1 (Plenum Press, New York 1988) p. 129

8. A.C. Engelsberg: In Surface Chemical Cleaning and Passivation for Semiconductor Processing, ed. by G.S. Higashi, E.A. Irene, T. Ohmi, Mater. Res. Soc. Proc. Vol. 315 (Pittsburgh, Penn., USA 1993) p. 255

9. Y.F. Lu, Y.W. Zheng, W.D. Song: J. Appl. Phys. 87, 2404 (2000)

10. P.K.H. Ho, D.S. Thomas, R.E. Friend, N. Tessler: Science 285, 233 (1999)

11. Y.F. Lu, Y.P. Lee, M.S. Zhou: J. Appl. Phys. 83, 1677 (1998)

12. K. Coupland, P.R. Herman, G. Bo: Appl. Surf. Sci. 127-129, 731 (1998)

13. M. Mosbacher, N. Chaoui, J. Siegel, V. Dobler, J. Solis, J. Boneberg, C.N. Afonso, P. Leiderer: Appl. Phys. A 69, 331 (1999)

14. S. Küper, J. Brannon, K. Brannon: Appl. Phys. A 56, 43 (1993)

15. M. Himmelbauer, E. Arenholz, D. Bäuerle: Appl. Phys. A 63, 87 (1996)

16. N. Arnold, N. Bityurin: Appl. Phys. A 68, 615 (1999)

17. G. Vereecke, E. Röhr, M.M. Heyns: J. Appl. Phys. 85, 3837 (1999)

18. B.D. Buckner, L. Suresh, E.D. Hirleman: Proc. SPIE 3275, 90 (1998)

19. M. Mosbacher, V. Dobler, J. Boneberg, P. Leiderer: Appl. Phys. A 70, 669 (2000)

20. P. Leiderer, J. Boneberg, V. Dobler, M. Mosbacher, H.J. Münzer, N. Chaoui, J. Siegel, J. Solis, C.N. Afonso, T. Fourrier, G. Schrems, D. Bäuerle: Proc. SPIE 4065 (2000)

21. S. Lazare, V. Granier: Laser Chem. 10, 25 (1989)

22. R. Srinivasan: In Interaction of Laser Radiation with Organic Polymers, ed. by J.C. Miller, Springer Series in Materials Science, Vol. 28 (Springer, Berlin, Heidelberg 1994) p. 107

23. V. Dobler, R. Oltra, J.P. Boquillon, M. Mosbacher, J. Boneberg, P. Leiderer: Appl. Phys. A 69(suppl.), 335 (1999)

24. Y.F. Lu, W.D. Song, B.W. Ang, M.H. Hong, D.S.H. Chan, R.S. Low: Appl. Phys. A 65, 9 (1997)

25. R. Srinivasan, B. Braren: Chem. Rev. 89, 1303 (1989)

26. D.S. Rimai, L.P. Demejo: Ann. Rev. Mater. Sci. 26, 21 (1996)

27. A.A. Kolomenskii, H.A. Schuessler, V.G. Mikhalevich, A.A. Maznev: J. Appl. Phys. 84, 2404 (1998)

28. Y.F. Lu, Y.W. Zheng, W.D. Song: Appl. Phys. A 68, 569 (1999)

29. M. Mosbacher, H.-J. Münzer, J. Zimmermann, J. Solis, J. Boneberg, P. Leiderer: Appl. Phys. A (2000), in press

30. O. Yavas, A. Schilling, J. Bischof, J. Boneberg, P. Leiderer: Appl. Phys. A 64, 331 (1997)

31. S. Boughaba, X. Wu, E. Sacher, M. Meunier: J. Adhes. 61, 293 (1997)

32. Y.F. Lu, Y. Zhang, W.D. Song, D.S.H. Chan: Jpn. J. Appl. Phys. 37, 1330 (1998) 